\title{
Iterative reconstruction methods in two different MDCT scanners: Physical metrics and 4-alternative forced-choice detectability experiments - A phantom approach
}

\author{
Frédéric A. Miéville ${ }^{\mathrm{a}, *}$, François Gudinchet ${ }^{\mathrm{b}}$, Francis Brunelle ${ }^{\mathrm{c}}$, \\ François O. Bochud ${ }^{a}$, Francis R. Verdun ${ }^{a}$
}

a Institute of Radiation Physics, University Hospital Center and University of Lausanne, 1 Rue du Grand-Pré, 1007 Lausanne, Switzerland

${ }^{\mathrm{b}}$ Department of Radiology, University Hospital Center and University of Lausanne, 46 Rue du Bugnon, 1011 Lausanne, Switzerland

'Department of Radiology, Necker Children's Hospital and University Paris Descartes, 149 Rue de Sèvres, 75743 Paris, France

Received 25 August 2011; received in revised form 5 December 2011; accepted 11 December 2011 Available online 2 January 2012

\author{
KEYWORDS \\ MDCT; \\ Iterative \\ reconstruction; \\ ASIR; \\ VEO; \\ iDose ${ }^{4}$; \\ MTF; \\ NPS; \\ 4AFC
}

\begin{abstract}
This paper characterizes and evaluates the potential of three commercial CT iterative reconstruction methods (ASIR ${ }^{T M}, V^{2} O^{T M}$ and iDose $^{4}{ }^{(T M}$ ) ) for dose reduction and image quality improvement. We measured CT number accuracy, standard deviation (SD), noise power spectrum (NPS) and modulation transfer function (MTF) metrics on Catphan phantom images while five human observers performed four-alternative forced-choice (4AFC) experiments to assess the detectability of low- and high-contrast objects embedded in two pediatric phantoms. Results show that 40\% and 100\% ASIR as well as iDose ${ }^{4}$ levels 3 and 6 do not affect CT number and strongly decrease image noise with relative SD constant in a large range of dose. However, while ASIR produces a shift of the NPS curve apex, less change is observed with iDose ${ }^{4}$ with respect to FBP methods. With second-generation iterative reconstruction VEO, physical metrics are even further improved: SD decreased to $70.4 \%$ at $0.5 \mathrm{mGy}$ and spatial resolution improved to $37 \%\left(\mathrm{MTF}_{50 \%}\right)$. 4AFC experiments show that few improvements in detection task performance are obtained with ASIR and iDose ${ }^{4}$, whereas VEO makes excellent detections possible even at an ultra-low-dose $(0.3 \mathrm{mGy})$, leading to a potential dose reduction of a factor 3 to $7(67 \%-86 \%)$. In spite of its longer reconstruction time and the fact that clinical studies are still required to complete these results, VEO clearly confirms the tremendous potential of iterative
\end{abstract}

\footnotetext{
* Corresponding author. Tel.: +41796546728.

E-mail address: frederic.mieville@chuv.ch (F.A. Miéville).
} 
reconstructions for dose reduction in CT and appears to be an important tool for patient followup, especially for pediatric patients where cumulative lifetime dose still remains high.

(c) 2011 Associazione Italiana di Fisica Medica. Published by Elsevier Ltd. All rights reserved.

\section{Introduction}

Today, ionizing radiation from computed tomography (CT) scanners represents the greatest per capita medical exposure for the population of industrialized countries [1-6]. Although this growth is mainly attributed to the increasing number of CT examinations, CT dose per examination is still high and remains an important concern.

Recently, new reconstruction algorithms based on iterative approaches were launched to complete existing strategies already widespread as a way to reduce $\mathrm{CT}$ dose during patient examination (tube current modulation, automatic exposure control, high pitch values on dualsource CT, automated kV modulation, etc.) [7-10]. In 2008, General Electric (Milwaukee, WI, USA) introduced the first CT iterative reconstruction method commercially available for clinical applications, under the name of adaptive statistical iterative reconstruction $\left(A S I R^{T M}\right)$. ASIR uses a blend of filtered back-projection (FBP) images with iteratively reconstructed images in the raw data domain to reduce the image noise [11-14]. Two years later, Siemens (Erlangen, Germany) released an iterative reconstruction in image space (IRIS'M). Contrary to ASIR, IRIS is based on an iterative reconstruction loop in the image domain to speed up the reconstruction process [15-17]. In 2011, Philips commercialized the fourth version of its iterative method, which was called iDose ${ }^{4}$ (тм) . This method attempts to reduce image noise without modifying the noise power spectrum (NPS) in order to keep close to the noise texture of classical FBP images $[18,19]$. After noise removal in the raw data domain, an optimal anatomical model is used in the image domain to iteratively eliminate the quantum image noise and to maintain the appearance of full dose image. Toshiba (Tochigi, Japan) has also developed an iterative method, adaptive iterative dose reduction $\left(A^{\prime D R}{ }^{T M}\right)$, which is adaptive and automatically calculates the optimized number of iterations [20]. Recently, GE introduced a full iterative reconstruction or model-based iterative reconstruction (MBIR), under the commercial name of VEOTM. Unlike its first iterative reconstruction ASIR, VEO is a fully iterative method working in the raw data domain, which takes not only the data statistics into account but also the geometry of the machine itself by considering the voxel volumes of the scanned object, the focal spot size, the active area size of the detector, etc. Moreover, to enhance model precision of the CT scanner, complex mathematical formulations were determined to account for physical effects such as beam hardening, scatter and metal attenuation artifacts [20,21]. Due to its complexity and specific properties, VEO is currently only designed for acquisitions performed with the Discovery CT750 HD scanner (GE Healthcare, Waukesha, WI, USA) and important reconstruction time is still required to reconstruct CT images.
The number and variety of new reconstruction methods introduces the need of investigating their dose and image quality relationships. Therefore, the purpose of this work was to characterize three commercial iterative reconstructions, ASIR, VEO and iDose ${ }^{4}$, through standard physical metrics (CT number accuracy, standard deviation (SD), modulation transfer function (MTF) and noise power spectrum) measured on Catphan phantom images. To go a step beyond physical measurements, five human observers performed four-alternative forced-choice (4AFC) experiments under the signal-known-exactly (SKE) paradigm to assess the detectability of low- and high-contrast objects embedded in two pediatric phantoms. Simple contrast and signal-difference-to-noise ratio (SDNR) metrics were also calculated and compared to low-contrast $4 \mathrm{AFC}$ results.

\section{Materials and methods}

\section{Data acquisition and image reconstruction}

Images were acquired on two multi-detector CT (MDCT) scanners: (A) a 128-slice Discovery CT750 HD scanner and (B) a 128-slice Ingenuity CT (Philips Healthcare, Cleveland, $\mathrm{OH}$, USA). Both systems were equipped with 64-row detector arrays able to supply up to $40 \mathrm{~mm}$ collimation with a standard configuration of $64 \times 0.625 \mathrm{~mm}$. Standard physical metrics were measured from Catphan 600 phantom (Phantom Laboratory, Salem, NY, USA) images scanned at 80 and $120 \mathrm{kVp}$. Because dose reduction is a main concern for pediatric patients, 4AFC experiments were also performed on pediatric phantom images obtained at $80 \mathrm{kVp}$. Fig. 1 shows the two pediatric phantoms used in this study. Each phantom was composed of an inner module surrounded by a soft tissue-equivalent ring to mimic the X-ray attenuation of a 2- to 3-year-old child. The 3D low-contrast module (QRM-3DLC; QRM, Moehrendorf, Germany) contains a cylinder (20 $\mathrm{mm}$ in diameter, $25 \mathrm{~mm}$ length) and several spheres $(3,4,5,6,8 \mathrm{~mm}$ in diameter) used to produce a low-contrast relative to the background of about $10 \mathrm{HU}$. The high-contrast structure module (QRM-CCl; QRM, Moehrendorf, Germany) is composed of three groups of small, radially arranged, cylindrical calcifications (density 200, 400 and $800 \mathrm{mg}$ hydroxyapatite $\left.(\mathrm{HA}) / \mathrm{cm}^{3}\right)$.

For all acquisitions but one, only the tube current was varied to get the $C T$ dose index $\left(C T D I_{v o l}\right)$ in the range 0.2-7.2 mGy. The exception was for unit B, where a lower pitch value had to be used to reach the maximum $C T D I_{\text {vol }}$ value. No automatic exposure control systems were employed. The scan and reconstruction parameters used on both CT units correspond to those of routine thorax protocols employed in our centers. The protocol parameters used in this study are presented in Table 1 . Note that protocols for CT units $A$ and $B$ are really close but are not identical due to $\mathrm{CT}$ unit specificities. 

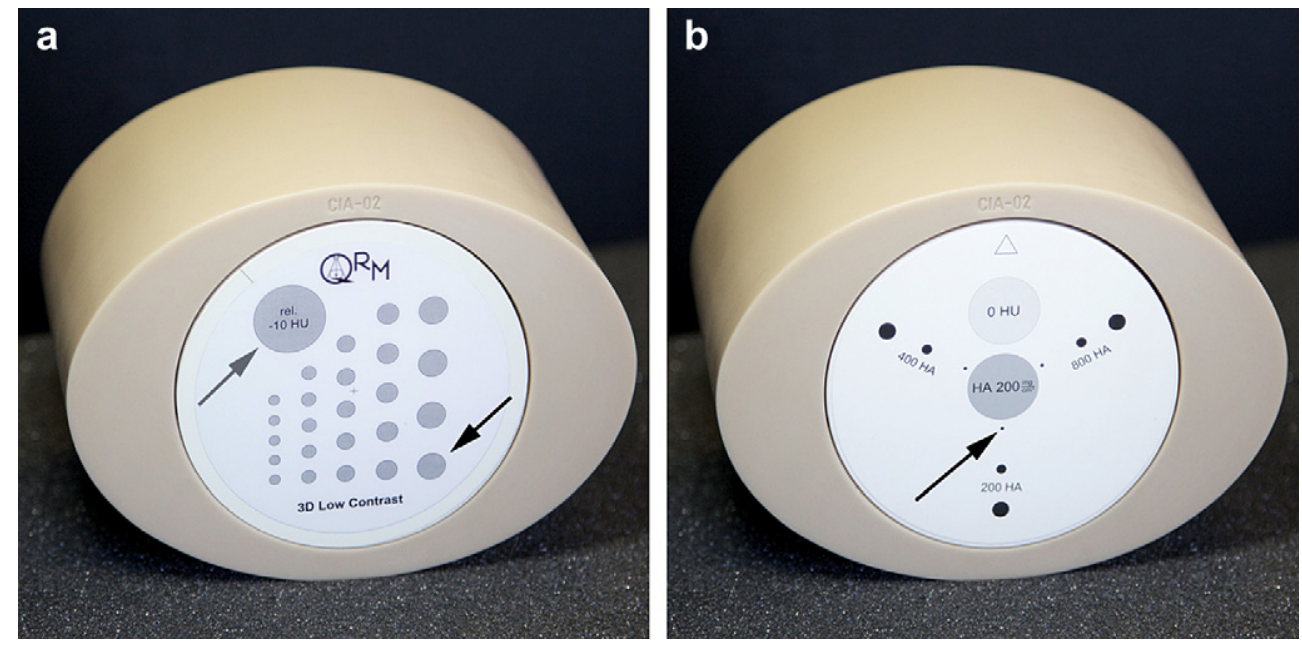

Figure 1 Pediatric phantoms used in this study. (a) Phantom made of a 3D low-contrast module surrounded by an equivalent tissue ring. (b) Phantom made of three groups of three cylindrical calcification inserts surrounded by an equivalent tissue ring. Phantom sizes correspond approximately to a 2-3 year-old child. Black arrows show the low- and high-contrast objects used for the $4 \mathrm{AFC}$ detectability experiments, mainly a low-contrast sphere $(8 \mathrm{~mm}$ in diameter with a relative contrast of about $-10 \mathrm{HU})$ and a microcalcification ( $1 \mathrm{~mm}$ length and $1 \mathrm{~mm}$ in diameter, density $200 \mathrm{mg} \mathrm{HA} / \mathrm{cm}^{3}$ ). The gray arrow shows the low-contrast cylinder ( $20 \mathrm{~mm}$ in diameter, $25 \mathrm{~mm}$ length with a relative contrast of about $-10 \mathrm{HU}$ ) used for contrast-to-noise measurements.

\section{Dosimetry}

$\mathrm{CTDI}_{\text {vol }}$ displayed at the CT consoles were verified by measuring the $\mathrm{CTDI}_{\text {vol }}$ in a $16 \mathrm{~cm}$ and $32 \mathrm{~cm}$ diameter CTDI phantoms with a $10 \mathrm{~cm}$ long CT pencil ion chamber connected to an electrometer (Radcal 1035-10.3 CTDI chamber, MDH 1015 electrometer; Radcal, Monrovia, CA). The pencil chamber and electrometer were calibrated in RQR9 and RQA9 beams according to IEC 61267 [22], and are traceable to the National Physical Laboratory. For each set of acquisition parameters, CTDI $_{\text {vol }}$ was calculated according to its definition $[23,24]$.

\section{Physical metrics}

CT number accuracy of various materials (air, PMP, Polystryrene, Delrin ${ }^{\mathrm{TM}}$ and Teflon) was measured from the Catphan sensitometry module (CTP 404). For each parameter condition $\left(\mathrm{CTDI}_{\mathrm{vol}}\right.$ and reconstruction methods), measurements were performed using a region of interest (ROI) of approximately $0.3 \mathrm{~cm}^{2}(14 \times 14$ pixels $)$ located at the center of the material insert. To obtain accurate results, the reported Hounsfield unit ( $\mathrm{HU}$ ) value was computed from 10 consecutive images and was expressed as the mean \pm standard error of the mean [25].

The noise value properties were characterized from Catphan uniform module (CTP 485) images using the pixel SD and NPS metrics. For each condition, SD was calculated from 30 replicated images using a ROI of $15 \mathrm{~cm}^{2}(100 \times 100$ pixels) located at the phantom center. The NPS analysis was computed from the 2D fast Fourier transform (FFT) operation using the following equation:

$\operatorname{NPS}\left(f_{x}, f_{y}\right)=\frac{b_{x} b_{y}}{L_{x} L_{y}}\left\langle\left|\operatorname{FFT}_{2 \mathrm{D}}\left\{\operatorname{ROI}(\boldsymbol{x}, \boldsymbol{y})-\mathrm{ROI}_{\text {background }}(\boldsymbol{x}, \boldsymbol{y})\right\}\right|^{2}\right\rangle_{\mathrm{N}_{\mathrm{ROI}}}$ where $b_{x}$ and $b_{y}$ are the sampling distances $(0.39 \mathrm{~mm})$ in the $x-$, and $y$-direction, respectively, and $L_{x}$ and $L_{y}$ are the ROI lengths in pixels (128 pixels) in the $x$ - and $y$-direction. $\mathrm{ROI}_{\text {background }}(x, y)$ is the background or structured noise measured from $\operatorname{ROI}(x, y)$ using a first-order (subtraction of a $3 \mathrm{D}$ linear fit) detrending technique. $N_{\mathrm{RO}}$ represents the number of ROls used in the average operation, noted $\langle\cdot\rangle$. For each reconstructed image, four ROls of $25 \mathrm{~cm}^{2}$ $(128 \times 128$ pixels $)$ each were extracted. Each ROI overlaps its direct neighbors with 32 pixels in the horizontal and vertical directions [26]. To reduce statistical fluctuations, 30 replicated images were used to extract 120 ROIs $(4 \times 30)$ and produce $\operatorname{NPS}\left(f_{x}, f_{y}\right)$. When computed at the phantom isocenter, $\operatorname{NPS}\left(f_{x}, f_{y}\right)$ has a rotational symmetry. Thus, to ease comparison of the NPS results and reduce the statistical fluctuations, a 1D NPS curve was determined. This operation was simply obtained by averaging the $\operatorname{NPS}\left(f_{x}, f_{y}\right)$ values corresponding to the same radial frequency $f_{r}$ [26]. Finally, NPS $\left(f_{r}\right)$ was fitted using a high-order polynomial (11order) to remove the fluctuations without modifying the NPS shape. The NPS results were verified by comparing the NPS area to the mean variance (square of noise) measured in the ROls. While this approach is consistent with current literature [27], it is worth mentioning that NPS measurements in CT are still a subject of ongoing research [AAPM task group, TG169].

The transverse spatial resolution was characterized through the in-plane MTF. To calculate this function, a ROI of approximately $9.8 \mathrm{~cm}^{2}(80 \times 80$ pixels $)$, centered on the bead point object image of the Catphan point source module (CTP 528), was selected. The ROI background was subtracted and the point-spread-function was integrated to yield the line-spread-function. Then, the MTF of the reconstructed image was calculated from the 1D FFT operation and normalized by the zerofrequency [26]. 
Table 1 Standard thorax protocols used for CT units A and B.

\begin{tabular}{|c|c|c|c|c|c|c|}
\hline \multirow[t]{2}{*}{ CT parameters } & \multicolumn{2}{|c|}{ Protocol 80kVp - Catphan } & \multicolumn{2}{|c|}{ Protocol $120 \mathrm{kVp}$ - Catphan } & \multicolumn{2}{|c|}{ Protocol $80 k V p-$ Ped phantoms } \\
\hline & Unit A & Unit B & Unit A & Unit B & Unit A & Unit B \\
\hline Voltage (kVp) & 80 & 80 & 120 & 120 & 80 & 80 \\
\hline $\begin{array}{l}\text { Detector config. } \\
(\text { rows } \times \mathrm{mm})\end{array}$ & $32 \times 1.25$ & $32 \times 1.25$ & $32 \times 1.25$ & $32 \times 1.25$ & $32 \times 1.25$ & $32 \times 1.25$ \\
\hline Scan field of view & Medium Body & - & Large Body & - & Medium Body & - \\
\hline $\mathrm{CTDI}_{\mathrm{vol}}(\mathrm{mG})^{*}$ & $0.5 / 1.0 / 2.0 / 4.1 / 7.1$ & $0.5 / 1.0 / 2.0 / 4.1 / 7.1$ & $0.6 / 1.1 / 2.1 / 3.9 / 7.2$ & $0.6 / 1.1 / 2.1 / 3.9 / 7.2$ & $0.2 / 0.3 / 0.4 / 1.0 / 2.0 / 7.1$ & $\begin{array}{l}0.2 / 0.3 / 0.4 / 1.0 \\
/ 2.0 / 7.1\end{array}$ \\
\hline $\begin{array}{l}\text { Grantry rotation } \\
\text { time }(\mathrm{sec})\end{array}$ & 0.5 & 0.5 & 0.5 & 0.5 & 0.5 & 0.5 \\
\hline $\begin{array}{l}\text { Tube current-time } \\
\text { product (mAs) }\end{array}$ & $\begin{array}{l}\text { Adjusted to } \\
\text { get required } \mathrm{CTDI}_{\mathrm{vol}}\end{array}$ & $\begin{array}{l}\text { Adjusted to } \\
\text { get required } \mathrm{CTDI}_{\mathrm{vol}}\end{array}$ & $\begin{array}{l}\text { Adjusted to } \\
\text { get required } \mathrm{CTDI}_{\mathrm{vol}}\end{array}$ & $\begin{array}{l}\text { Adjusted to } \\
\text { get required } \mathrm{CTDI}_{\mathrm{vol}}\end{array}$ & $\begin{array}{l}\text { Adjusted to } \\
\text { get required } \mathrm{CTDI}_{\mathrm{vol}}\end{array}$ & $\begin{array}{l}\text { Adjusted to } \\
\text { get required } \mathrm{CTDI}_{\mathrm{vol}}\end{array}$ \\
\hline Acquisition mode & Helical & Helical & Helical & Helical & Helical & Helical \\
\hline Pitch & 1.375 & $1.344(0.718)^{* *}$ & 1.375 & $1.344(0.718)^{* *}$ & 1.375 & $1.344(0.718)^{* *}$ \\
\hline \multirow{4}{*}{$\begin{array}{l}\text { Reconstruction } \\
\text { algorithm }\end{array}$} & FBP & FBP & FBP & FBP & FBP & FBP \\
\hline & $40 \%$ ASIR & iDose $^{4}$ L3 & $40 \%$ ASIR & iDose ${ }^{4}$ L3 & $100 \%$ ASIR & iDose $^{4}$ L6 \\
\hline & $100 \%$ ASIR & iDose ${ }^{4}$ L6 & $100 \%$ ASIR & iDose $^{4}$ L6 & & \\
\hline & VEO & & VEO & & VEO & \\
\hline Reconstruction plane & Axial & Axial & Axial & Axial & Axial & Axial \\
\hline $\begin{array}{l}\text { Recon. slice } \\
\text { thickness (mm) }\end{array}$ & 1.25 & 1.5 & 1.25 & 1.5 & 1.25 & 1.5 \\
\hline Recon. interval (mm) & 1.25 & 1.5 & 1.25 & 1.5 & 1.25 & 1.5 \\
\hline Reconstruction filter & Standard & B (standard) & Standard & B (standard) & Standard & B (standard) \\
\hline $\begin{array}{l}\text { Display field of } \\
\text { view }(\mathrm{mm})\end{array}$ & 200 & 200 & 200 & 200 & 130 & 130 \\
\hline Matrix size & $512 \times 512$ & $512 \times 512$ & $512 \times 512$ & $512 \times 512$ & $512 \times 512$ & $512 \times 512$ \\
\hline High resolution mode & not used & not used & not used & not used & not used & not used \\
\hline
\end{tabular}


Prior to the 4AFC detectability experiments, the contrast, noise and SDRN were analyzed using the lowcontrast cylinder embedded in the 3D low-contrast pediatric phantom (Fig. 1a). A ROI of $1.56 \mathrm{~cm}^{2}(32 \times 32$ pixels) was determined inside the cylinder, and four smaller ROI $(12 \times 12$ pixels) were identified surrounding the cylinder. The contrast, $C$, was defined as:

$C=\left|\mathrm{HU}_{\text {sphere }}-\mathrm{HU}_{\text {background }}\right|$

where $\mathrm{HU}_{\text {sphere }}$ and $\mathrm{HU}_{\text {background }}$ corresponds to the average CT number of the sphere and background, respectively.

The average noise, $\mathrm{SD}_{\mathrm{sph} / \mathrm{bkgd}}$, of the sphere and background was defined as:

$\mathrm{SD}_{\text {sph } / \text { bkgd }}=\left(\mathrm{SD}_{\text {sphere }}+\mathrm{SD}_{\text {background }}\right) / 2$

where $\mathrm{SD}_{\text {sphere }}$ and $\mathrm{SD}_{\text {background }}$ corresponds to the average noise of the sphere and background, respectively.

Finally, the signal-difference-to-noise ratio, or contrastto-noise ratio (CNR), was computed as:

$\mathrm{SDNR}_{\text {sph } / \text { bkgd }}=\frac{C}{\mathrm{SD}_{\text {sph } / \text { bkgd }}}=\frac{\left|\mathrm{HU}_{\text {sphere }}-\mathrm{HU}_{\text {background }}\right|}{\left(\mathrm{SD}_{\text {sphere }}+\mathrm{SD}_{\text {background }}\right) / 2}$

Results were obtained using eight consecutive slices and were expressed as the mean \pm standard error of the mean.

\section{AFC detectability experiments}

Four 4AFC experiments were set up: (i) low-contrast object detectability (on image acquired and reconstructed) on unit A, (ii) low-contrast object detectability on unit B, (iii) high-contrast object detectability on unit $A$ and (iv) highcontrast object detectability on unit $B$.

The methodology used to build the 4AFC experiments is illustrated using the low-contrast sphere experiment performed on unit A. Dedicated programs were created in MATLAB R2011a (Mathworks, Natick, MA, USA) to automatically execute the following steps: first, from the images obtained at the highest dose $(7.1 \mathrm{mGy})$ and reconstructed with the FBP methods, we selected the 2 slices where the sphere object was the most visible as well as the 4 following slices. This enabled us to extract the 2 object images (ROIs) and 6 background images (on background per slice) and generated a stack of eight images, all with a size of $100 \times 100$ pixels. Note that to obtain a background texture close to the one of the background object, background images were chosen in the vicinity of the object but far enough away to avoid the influence of the latter on the background noise. Keeping ROI positions and sizes constant, the procedure was repeated to build the 8 -image stacks corresponding to the different reconstruction and dose conditions. For unit A, 18 stacks ( 3 reconstructions $\times 6$ doses) were obtained. Then, 1 object and 3 backgrounds were randomly selected from each stack. The four selected images were arbitrarily rotated at $90^{\circ}, 180^{\circ}$ or $270^{\circ}$ before being randomly displayed in one of the four possible locations of the $4 \mathrm{AFC}$ trial. Experiment (i) was composed of 180 trials (10 repetitions of the 18 stacks) conducted in random order.

The same methodology was applied to build Experiment (ii) and led to 12 stacks ( 2 reconstructions $\times 6$ doses) and 120 trials (10 repetitions of the 12 stacks). Experiments (iii) and (iv) associated to the detectability of the microcalcifications were built in the same way as Experiments (i) and (ii), however, due to the small size of the object $(1 \mathrm{~mm}$ length and $1 \mathrm{~mm}$ in diameter), the ROI size of the object was smaller $(75 \times 75$ pixels $)$ and only one slice containing the object was selected. This led to 7-image stacks instead of 8-image stacks.

Figure $2 \mathrm{a}$ and $\mathrm{b}$ illustrate two trails obtained from Experiments (i) and (iii) when images were acquired at 7.1 $\mathrm{mGy}$ and reconstructed with the FBP method. Lowcontrast sphere and microcalcification objects are located at positions 2 and 4 , respectively.

Five trained naïve (non-physician) observers participated in 4AFC detectability experiments. The training set involved an additional 20 trials presented prior to each experiment. With the SKE display at reference, the observers had to determine which image was the most likely to contain the signal by clicking on it. Viewing time was not limited and no direct feedback was provided after each trial. The images were displayed in a low light environment on a LED monitor (SyncMaster XL2370 monitor, Samsung, Inc.). For each trial, window settings were automatically adjusted based on the maximal and minimal $\mathrm{HU}$ values of the images and then kept identical for the 4 images. The magnification was fixed and the observers were only free to adjust the viewing distance (typically corresponding to $\sim 40 \mathrm{~cm}$ ).

The percentage correct $(\mathrm{PC})$, corresponding to the percentage of correct answers given by an observer for a 4AFC experiment performed in a defined condition, was analyzed as a figure of merit of object detectability and evaluated as a function of CTDI $_{\mathrm{vol}}$. PC $=100 \%$ correlates to

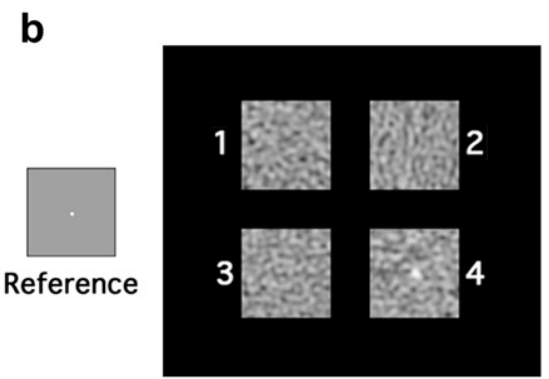

Figure 2 Illustration of the 4AFC detectability experiments. (a) The low-contrast sphere and (b) the high-contrast cylindrical calcification when images are reconstructed with the FBP method on CT unit A. Objects are visible in position 2 and 4 , respectively. Acquisitions were performed at $80 \mathrm{kVp}$ and $7.1 \mathrm{mGy}$. 
Table 2 Normalized weighted ${ }_{n} \mathrm{CTDI}_{\mathrm{w}}$ for unit A. Acquisitions were performed in axial mode at $80 \mathrm{kVp}$.

\begin{tabular}{lllll}
\hline CT unit & Phantom diameter $\mathrm{kVp}$ & Measured $_{\mathrm{n}} \mathrm{CTDI}_{\mathrm{w}}(\mathrm{mGy} / 100 \mathrm{mAs})$ & Unit $_{\mathrm{n}} \mathrm{CTDI}_{\mathrm{w}}(\mathrm{mGy} / 100 \mathrm{mAs})$ & Relative difference $(\%)$ \\
\hline $\mathrm{A}$ & $\varnothing 32 \mathrm{~cm}, 120 \mathrm{kVp}$ & 7.62 & 8.56 & 11 \\
& $\varnothing 32 \mathrm{~cm}, 80 \mathrm{kVp}$ & 2.28 & 2.65 & 14 \\
& $\varnothing 16 \mathrm{~cm}, 80 \mathrm{kVp}$ & 4.66 & $5.30^{\mathrm{a}}$ & 12 \\
\hline
\end{tabular}

a Unit ${ }_{n} \mathrm{CTDI}_{\mathrm{W}}$ multiplied by 2 .

a perfect response while $P C=25 \%$ implies pure guessing for 4 AFC experiments. On each PC versus $C_{T D I}$ vol plot, the resulting $P C$ was fitted with one-parameter curve, $\mathrm{PC}=100 \cdot\left(1-e^{-\alpha \cdot C T D I}\right)$ to provide a visual guide, and not meant to represent a physical model.

\section{Results}

The normalized weighted CTDI $\left({ }_{n} \mathrm{CTDI}_{\mathrm{w}}\right)$ measured at $80 \mathrm{kVp}$ and $120 \mathrm{kVp}$ in the $32 \mathrm{~cm}$ CTDI test phantom and at $80 \mathrm{kVp}$ in the $16 \mathrm{~cm}$ phantom are presented in Tables 2 and 3. A good agreement $(<14 \%)$ between console displays and measured values was obtained for both CT units.

\section{Physical metrics}

Figure 3 shows Catphan sensitometry images obtained from the same scan when reconstructed with the 3 different methods of unit A; FBP, $100 \%$ ASIR and VEO. While image noise was strongly reduced and the perceived visibility of structures was slightly improved with $100 \%$ ASIR, VEO even further decreased SD and clearly improved the visibility of the Catphan beads and targets, especially the acrylic one. However, with this latter reconstruction, the noise texture was less grainy than the ones obtained with $100 \%$ ASIR or FBP, according to the radiologists' subjective points of view. Fig. 4 shows sensitometry images obtained on unit $B$. An important noise reduction and an improvement of the overall image quality were observed with iDose ${ }^{4}$ level 6 (L6) in comparison to FBP.

Impact of the iterative reconstructions on CT number accuracy was verified on images acquired at 80 and $120 \mathrm{kVp}$ for both CT units. Tables 4 and 5 report the measured CT number values of several materials present in the sensitometry module. The results show that $100 \%$ ASIR, VEO, and iDose ${ }^{4}$ L6 do not significantly modify the HU values with respect to the standard reconstruction methods.

The noise mean value was assessed through SD measurements on images acquired at $80 \mathrm{kVp}$. Figs. $5 \mathrm{a}$ and 6a show SDs as a function of CTDI $_{\text {vol }}$ for the different reconstructions available on $C T$ units $A$ and $B$. As expected, important decreases of noise values were reached with the iterative reconstructions in comparison with the associated FBP methods. SD values measured at 0.5 and $7.1 \mathrm{mGy}$ are summarized in the tables included in both figures. For unit $A$, relative differences between iterative and FBP noise values are $20 \%, 47 \% 70 \%$ at $0.5 \mathrm{mGy}$ and $22 \%, 48 \%, 43 \%$ at $7.1 \mathrm{mGy}$, for $40 \%$ ASIR, $100 \%$ ASIR and VEO, respectively. Results show that the benefit of ASIR is almost constant when $\mathrm{CTDI}_{\mathrm{vol}}$ varies in a large range of dose while with VEO an increase of the relative noise difference is observed when dose reduces. As expected, SD reduces as the inverse square root of dose for all the reconstructions as indicated by the fitting curves $\left(\sim \mathrm{CTDI}_{\mathrm{vol}}^{-1 / 2}\right)$. However a fitting curve of the form $\alpha \cdot \mathrm{CTDI}_{\mathrm{vol}}^{-1 / 2}+\beta$, where $\alpha$ and $\beta$ are constant parameters, had to be used to better represent the behavior of SD with VEO. For unit B, relative noise differences were $22 \%, 43 \%$ at $0.5 \mathrm{mGy}$ and $21 \%, 44 \%$ at $7.1 \mathrm{mGy}$ for iDose ${ }^{4}$ level $3(\mathrm{~L} 3)$ and iDose ${ }^{4}$ L6, respectively, leading to a constant benefit of iDose ${ }^{4}$ when $\mathrm{CTDI}_{\mathrm{vol}}$ reduces.

Figure $5 b$ and $6 b$ show the MTF curves obtained from the Catphan point source bead scanned at $4.1 \mathrm{mGy}$ and images reconstructed with the FBP and iterative reconstruction methods. For unit A, the frequency values extracted at an MTF level of $50 \%\left(M_{T F} F_{50 \%}\right)$ are $0.35,0.36,0.37$ and $0.48 \mathrm{~mm}^{-1}$, for FBP, 40\% ASIR, 100\% ASIR and VEO, respectively. This corresponds to relative spatial frequency improvements of $3 \%, 6 \%$ and $37 \%$ when using $40 \%$ ASIR, $100 \%$ ASIR and VEO with respect to FBP. For unit B, no frequency improvements were measureable between FBP and iDose ${ }^{4}$ and $\mathrm{MTF}_{50 \%}$ value was $0.33 \mathrm{~mm}^{-1}$. Note that similar results were observed at $7.1 \mathrm{mGy}$ with a pitch value of 0.718 .

Figures $5 c$ and $6 c$ show the noise amplitude as a function of the spatial frequency. NPS curves were calculated from the homogenous module images scanned at $80 \mathrm{kVp}$ and $1 \mathrm{mGy}$. The analysis of the NPS curves obtained with FBP shows that the reconstruction kernels of unit $A$ and $B$ have different shapes (depending on the manufacturer' strategy). For unit A, the apexes of the NPS curves are located at $0.32,0.26,0.19$ and $0.13 \mathrm{~mm}^{-1}$ for FBP, $40 \%$ ASIR, 100\% ASIR and VEO, respectively, and correspond to a relative shift of the apexes of $21 \%, 41 \%$ and $61 \%$ when compared to FBP (Fig. 5d). For unit B, the apexes of the NPS

Table 3 Normalized weighted ${ }_{n} \mathrm{CTDI}_{\mathrm{w}}$ for unit B. Acquisitions were performed in axial mode at $80 \mathrm{kVp}$.

\begin{tabular}{lllll}
\hline CT unit & Phantom diameter $\mathrm{kVp}$ & Measured ${ }_{n} \mathrm{CTDI}_{\mathrm{w}}(\mathrm{mGy} / 100 \mathrm{mAs})$ & Unit ${ }_{\mathrm{n}} \mathrm{CTDI}_{\mathrm{w}}(\mathrm{mGy} / 100 \mathrm{mAs})$ & Relative difference $(\%)$ \\
\hline $\mathrm{B}$ & $\emptyset 32 \mathrm{~cm}, 120 \mathrm{kVp}$ & 6.32 & 6.56 & 4 \\
& $\varnothing 32 \mathrm{~cm}, 80 \mathrm{kVp}$ & 1.75 & 1.86 & 6 \\
& $\varnothing 16 \mathrm{~cm}, 80 \mathrm{kVp}$ & 3.61 & $3.72^{\mathrm{a}}$ & 3 \\
\hline
\end{tabular}

${ }^{a}$ Unit ${ }_{n} \mathrm{CTDI}_{\mathrm{W}}$ multiplied by 2 . 


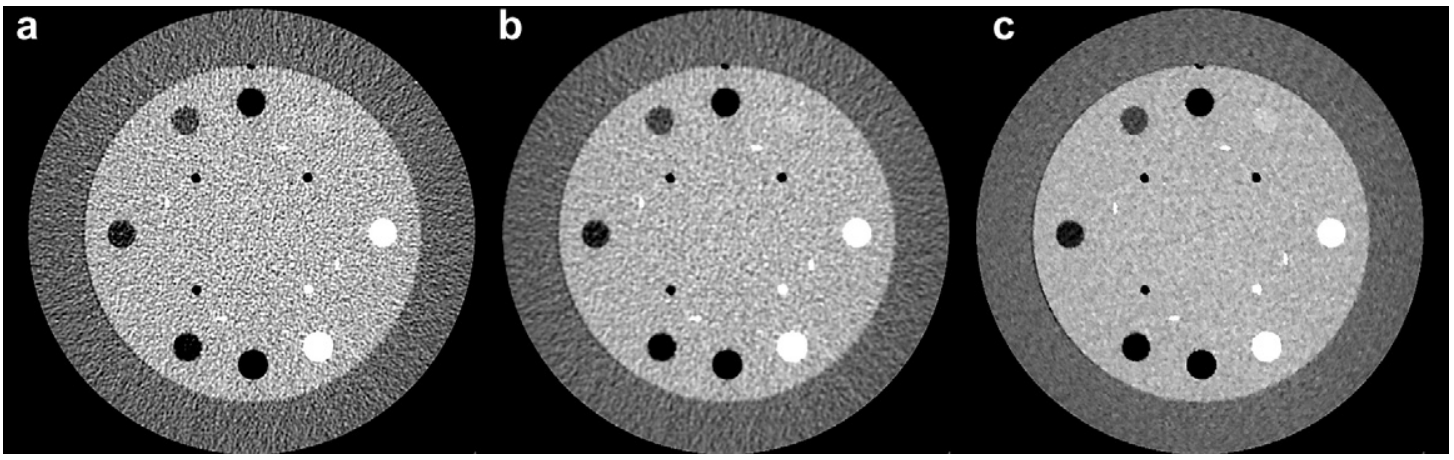

Figure 3 Catphan sensitometry module scanned at $120 \mathrm{kVp}$ and $1.0 \mathrm{mGy}$ on unit A. Reconstruction methods are: (a) FBP (unit A), (b) $100 \%$ ASIR and (c) VEO. From top and in the clockwise direction, air, acrylic, Delrin ${ }^{\mathrm{TM}}$, Teflon, air, PMP, LDPE and polystyrene targets are visible.

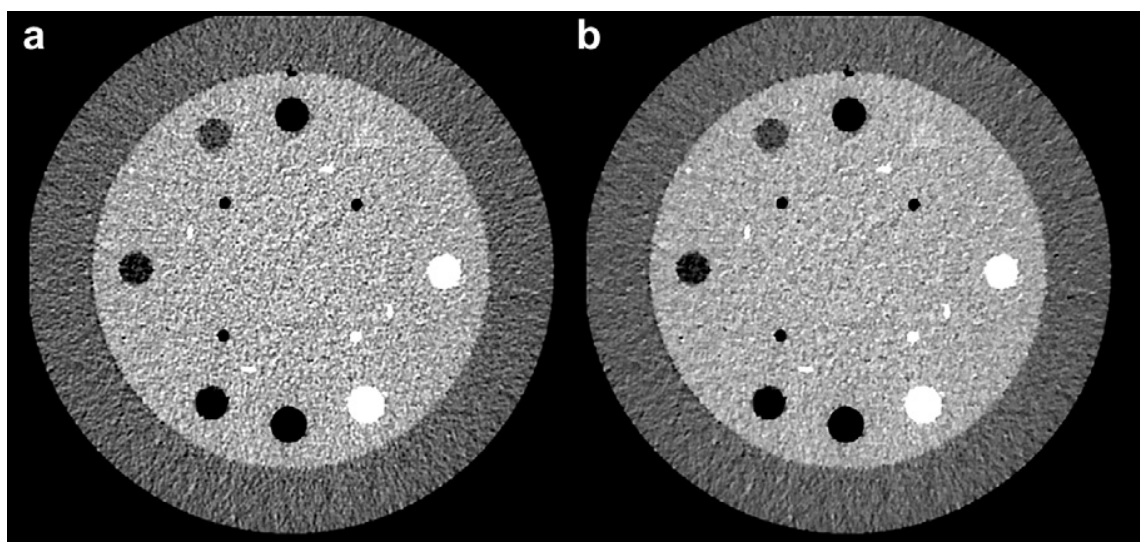

Figure 4 Catphan sensitometry module scanned at $120 \mathrm{kVp}$ and $1.0 \mathrm{mGy}$ on unit B. Reconstruction methods are: (a) FBP (unit B) and (b) iDose ${ }^{4}$ L6.

curves were located at $0.24,0.20$ and $0.17 \mathrm{~mm}^{-1}$ for FBP, iDose $^{4} \mathrm{~L} 3$ and iDose ${ }^{4} \mathrm{~L} 6$, respectively, and associated relative shifts were $17 \%$ and $29 \%$ (Fig. 6d). It is worth pointing out that the area under the NPS curve corresponds to the square of SD within computing uncertainties. At $120 \mathrm{kVp}$ and for various $\mathrm{CTDI}_{\text {vol }}$, NPS curves show the same behavior than the one obtained at $80 \mathrm{kVp}$ and $1 \mathrm{mGy}$.

Because the $8 \mathrm{~mm}$ sphere was not large enough to accurately measure the contrast, average noise and SDNR, measurements were performed for the low-contrast cylinder (both objects have the same relative contrast, $-10 \mathrm{HU})$.
While the contrast was independent of $\mathrm{CTDI}_{\text {vol }}$ (within experimental error) on both units, the noise and SDNR varied as a function of dose as shown in Figs. 7 and 8. For unit A, $\mathrm{SD}_{\mathrm{sph} / \mathrm{bkgd}}$ reduces at the inverse root of $\mathrm{CTDI}_{\mathrm{vol}}$ for FBP and $100 \%$ ASIR, however, a different behavior is obtained with

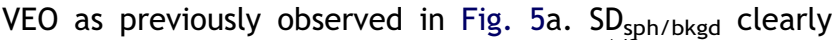
moves away from its fitting curve, $\sim \mathrm{CTDI}_{\mathrm{vol}}^{-1 / 2}$, and appears to be less sensitive to the dose. Because the contrast is constant, a similar effect is visible for SDNR when plotted as

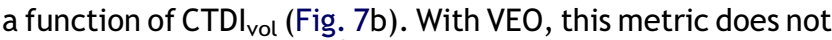
follow the fit $\sim \mathrm{CTDI}_{\mathrm{vol}}^{1 / 2}$ contrary to results with FBP and $100 \%$ ASIR, which increase at the root of the dose.

Table 4 Mean CT number measured on the Catphan sensitometry module when scanned on unit A for CTDI ${ }_{\mathrm{vol}}=1.0 \mathrm{mGy}$. Values are based on the measurements of 10 consecutive images.

\begin{tabular}{|c|c|c|c|c|c|c|}
\hline \multirow[b]{2}{*}{$\mathrm{kVp}$} & \multirow[b]{2}{*}{ Algorithm } & \multicolumn{5}{|l|}{ Insert material } \\
\hline & & Air $\approx-1000 \mathrm{HU}$ & $\mathrm{PMP} \approx-200 \mathrm{HU}$ & Poly $\approx-35 \mathrm{HU}$ & Delrin $^{\mathrm{TM}} \approx 340 \mathrm{HU}$ & Teflon $\approx 990 \mathrm{HU}$ \\
\hline \multirow[t]{3}{*}{80} & FBP(unit A) & $-987.2 \pm 2.0$ & $-210.5 \pm 2.6$ & $-59.7 \pm 1.7$ & $337.2 \pm 4.8$ & $1020.4 \pm 7.8$ \\
\hline & $100 \%$ ASIR & $-989.7 \pm 2.4$ & $-210.8 \pm 2.4$ & $-60.0 \pm 1.6$ & $338.4 \pm 4.8$ & $1020.5 \pm 7.5$ \\
\hline & VEO & $-986.5 \pm 0.8$ & $-206.9 \pm 1.5$ & $-64.8 \pm 0.8$ & $334.9 \pm 2.4$ & $1010.1 \pm 3.9$ \\
\hline \multirow[t]{3}{*}{120} & FBP(unit A) & $-982.7 \pm 2.0$ & $-176.2 \pm 1.1$ & $-31.6 \pm 1.7$ & $357.5 \pm 4.4$ & $967.7 \pm 6.5$ \\
\hline & $100 \%$ ASIR & $-984.8 \pm 2.3$ & $-176.3 \pm 1.1$ & $-30.9 \pm 1.5$ & $357.2 \pm 4.2$ & $967.0 \pm 6.4$ \\
\hline & VEO & $-982.6 \pm 1.3$ & $-176.1 \pm 0.8$ & $-34.3 \pm 0.8$ & $353.8 \pm 2.8$ & $957.4 \pm 3.2$ \\
\hline
\end{tabular}


Table 5 Mean CT number measured on the Catphan sensitometry module when scanned on unit B for CTDI $\mathrm{vol}_{\mathrm{vo}}=1.0 \mathrm{mGy}$. Values are based on the measurements of 10 consecutive images.

\begin{tabular}{|c|c|c|c|c|c|c|}
\hline \multirow[b]{2}{*}{$\mathrm{kVp}$} & \multirow[b]{2}{*}{ Algorithm } & \multicolumn{5}{|l|}{ Insert material } \\
\hline & & Air $\approx-1000 \mathrm{HU}$ & $\mathrm{PMP} \approx-200 \mathrm{HU}$ & Poly $\approx-35 \mathrm{HU}$ & Delrin $^{\mathrm{TM}} \approx 340 \mathrm{HU}$ & Teflon $\approx 990 \mathrm{HU}$ \\
\hline \multirow[t]{2}{*}{80} & FBP(unit $B$ ) & $-992.4 \pm 1.2$ & $-215.5 \pm 1.2$ & $-60.9 \pm 2.7$ & $346.1 \pm 1.6$ & $1004.3 \pm 3.1$ \\
\hline & iDose L6 & $-995.4 \pm 1.1$ & $-215.7 \pm 1.0$ & $-60.3 \pm 2.3$ & $346.1 \pm 1.4$ & $1004.5 \pm 2.6$ \\
\hline \multirow[t]{2}{*}{120} & FBP(unit B) & $-979.8 \pm 1.9$ & $-177.5 \pm 1.6$ & $-26.1 \pm 1.7$ & $343.3 \pm 2.3$ & $949.2 \pm 1.7$ \\
\hline & iDose L6 & $-979.6 \pm 1.6$ & $-177.4 \pm 1.4$ & $-26.0 \pm 1.4$ & $343.4 \pm 2.1$ & $948.9 \pm 1.6$ \\
\hline
\end{tabular}

For unit $B$, the noise and SDNR obtained with iDose ${ }^{4}$ L6 are both improved when compared to FBP results with metric behaviors following a Poisson distribution as shown in Fig. 8.

\section{AFC detectability experiments.}

Figures 9-12 show the results of the 4AFC detectability experiments when $\mathrm{PC}$ is reported as a function of CTDI ${ }_{\mathrm{vol}}$. Error bars represent standard errors and correspond to the inter-observer variability.

\section{Low-contrast sphere object}

Figures 9 and 10 show PC for the low-contrast sphere object. As expected, the general trend is an increase of
PC with dose (within the experimental fluctuations). For unit $A$, the use of $100 \%$ ASIR provides a slightly higher PC in the detection of the low-contrast object while VEO clearly allows the detection of the object even at ultralow-doses as shown in Fig. 9. For $\mathrm{CTDI}_{\mathrm{vol}}$ from 1.0 to $0.3 \mathrm{mGy}$, the results indicate a significant decrease of PC $(\mathrm{PC}=\sim 70 \%)$ with $\mathrm{FBP}$ and $100 \%$ ASIR while VEO still provides perfect detectability $(P C=100 \%)$. At $\mathrm{CTDI}_{\mathrm{vol}}=0.2 \mathrm{mGy}$, the percentage of exact responses obtained with VEO drops to PC $=\sim 85 \%$. Fig. 10 shows $P C$ obtained with FBP and iDose ${ }^{4}$ L6 methods (unit B). As for $100 \%$ ASIR, a higher confidence level in the detection of the sphere was observed. However, no clear improvements in detection performances were noticed with iDose ${ }^{4}$ L6.
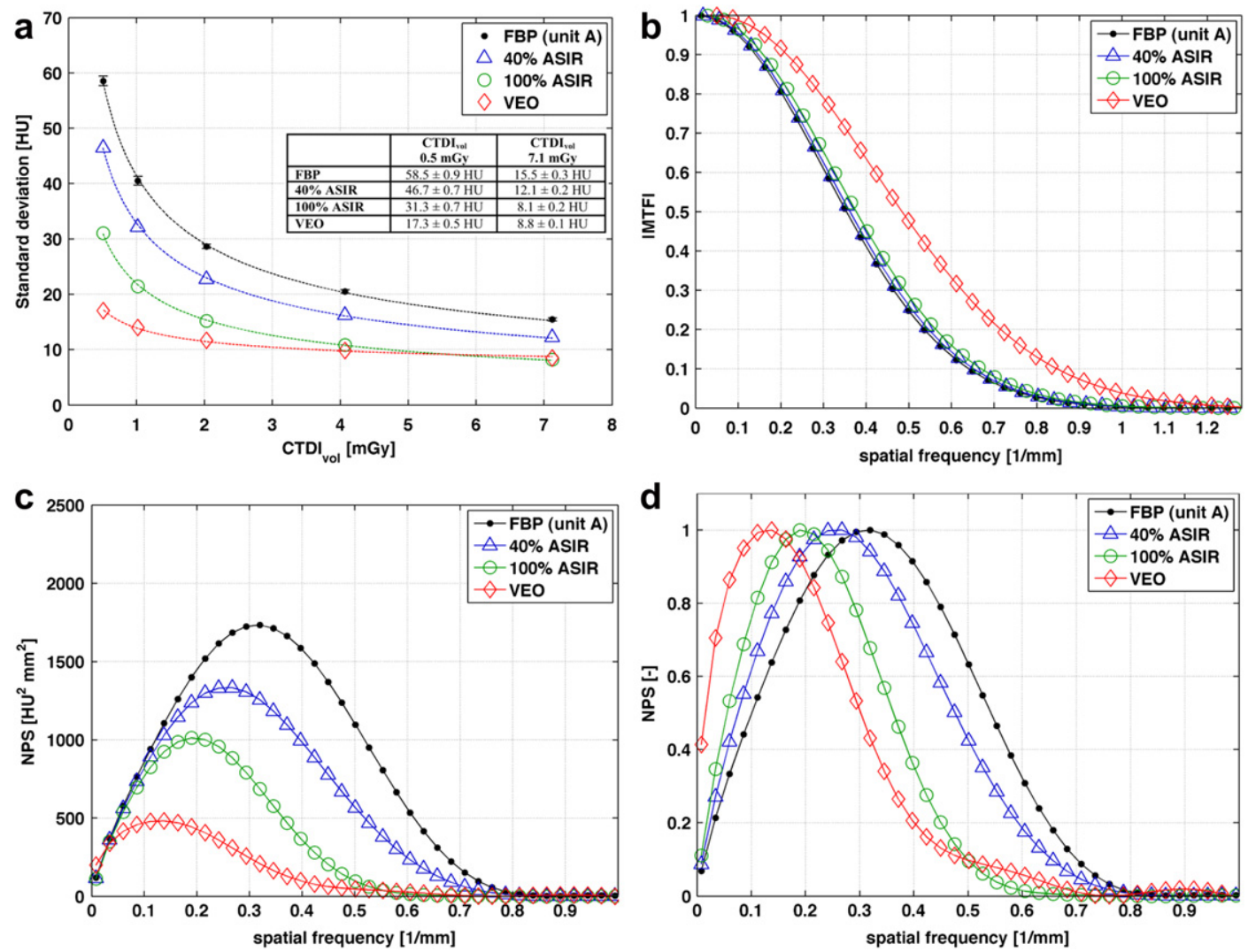

Figure 5 Physical metrics as a function of various reconstruction methods measured from the Catphan phantom at $80 \mathrm{kVp}$ on unit A. (a) SD as a function of CTDI ${ }_{\text {vol }}$. Fitting curves (dashed lines), $\sim$ CTDI $_{\text {vol }}^{-1 / 2}$, were used for FBP, $40 \%$ and $100 \%$ ASIR, while for VEO, the fitting curve was $\alpha \cdot \mathrm{CTDI}_{\mathrm{vol}}^{-1 / 2}+\beta$, where $\alpha$ and $\beta$ are constant parameters. (b) MTF computed from images obtained at $4.1 \mathrm{mGy}$ (c) NPS obtained at 1.0 mGy and (d) NPS normalized at the maximum NPS value. While important noise reduction is obtained with $40 \%$ and $100 \%$ ASIR, VEO allows further noise reduction with a significant improvement in spatial resolution. 

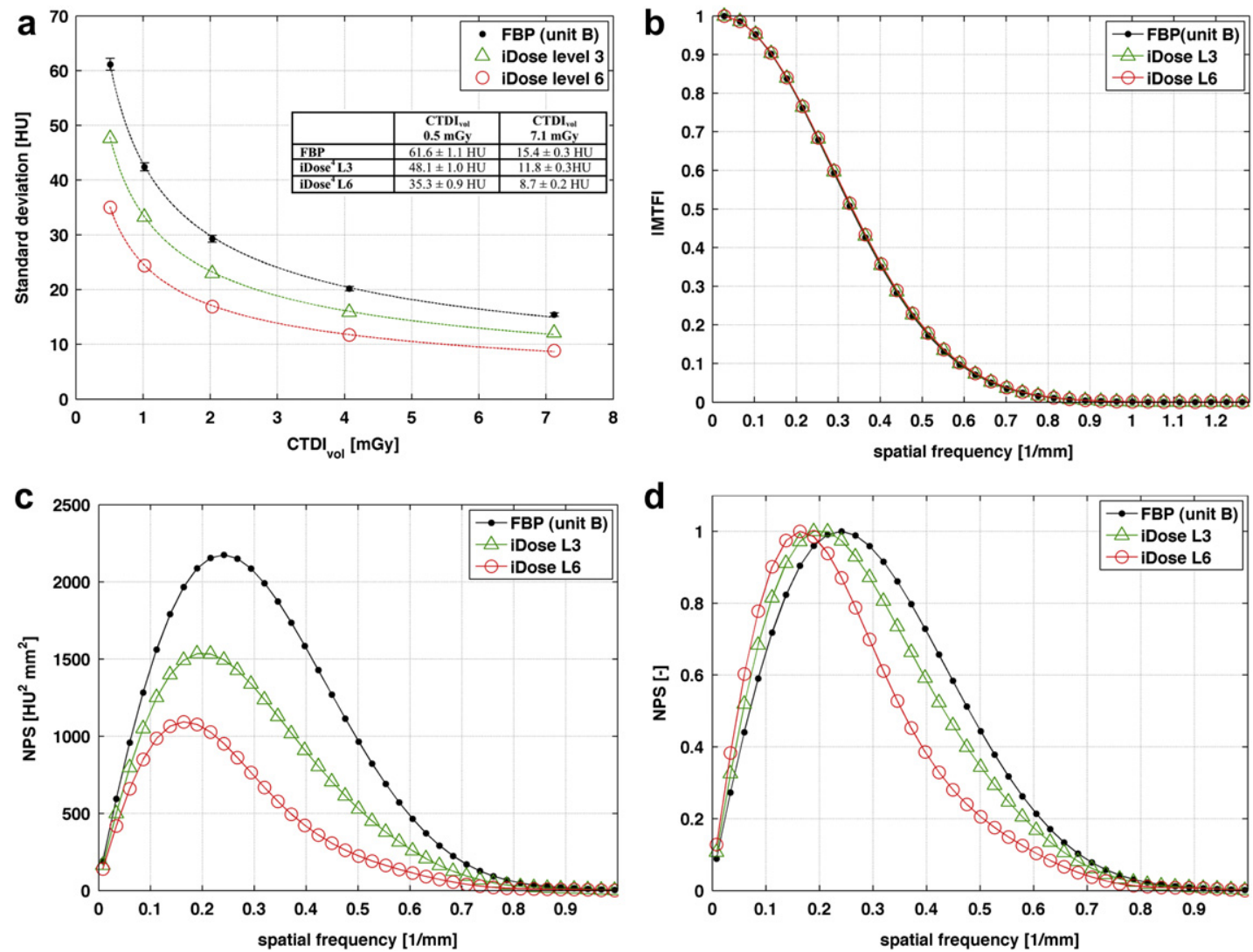

Figure 6 Physical metrics as a function of various reconstruction methods measured from the Catphan phantom at $80 \mathrm{kVp}$ on unit B. (a) SD as a function of CTDI ${ }_{\text {vol. }}$ Fitting curves (dashed lines), $\sim \mathrm{CTDI}_{\text {vol }}^{-1 / 2}$, were used for FBP and iDose ${ }^{4}$ level 3 and 6 . (b) MTF computed from images obtained at $4.1 \mathrm{mGy}$ (c) NPS obtained at $1.0 \mathrm{mGy}$ and (d) NPS normalized at the maximum NPS value. Important noise reduction without changes in MTF curves is obtained with iDose ${ }^{4}$.

\section{Microcalcification (high contrast) object}

The microcalcification detectability results are presented in Figs. 11 and 12. For unit A, the same general trend as the low-contrast sphere was observed with the microcalcification. VEO allows an excellent detection of the object up to $0.3 \mathrm{mGy}$ while a decrease is observed at $0.2 \mathrm{mGy}$. However, contrary to the sphere, no significant improvement was observed with $100 \%$ ASIR when compared to FBP.

Figure 12 shows the results of the 4 AFC detectability experiment obtained for the high-contrast object on unit $B$. Although the noise pixel is reduced with iDose ${ }^{4}$ L6, in this particular situation, no significant improvement was obtained with respect to the FBP reconstruction method. Note
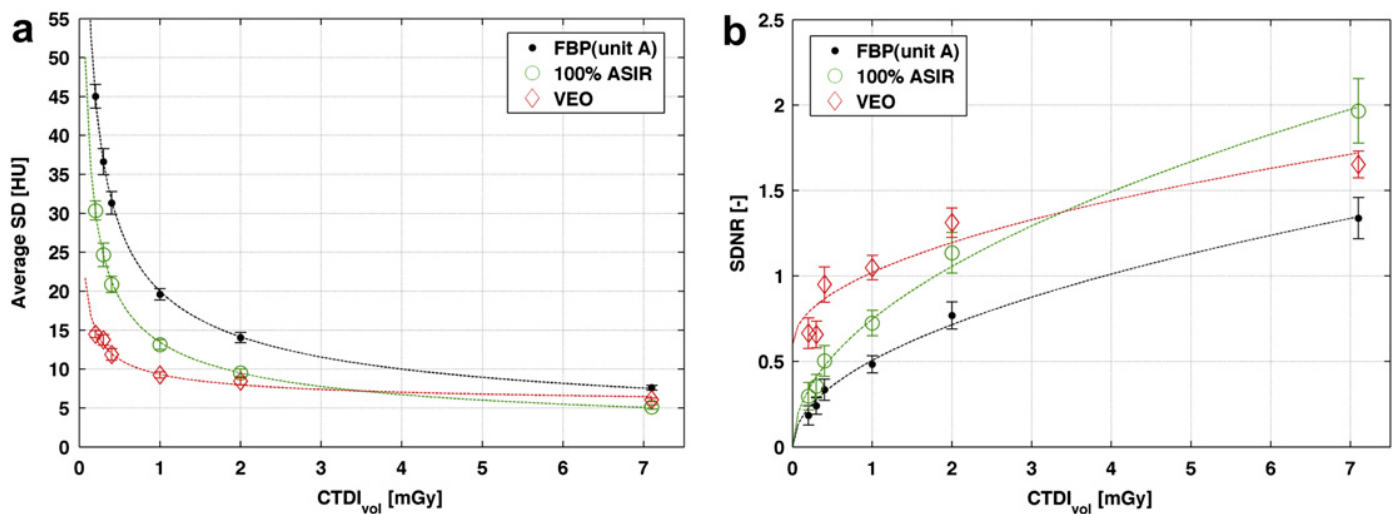

Figure 7 (a) Average SD and (b) SDNR measured on the low-contrast cylinder (Fig. 1a) as a function of dose on unit A. Acquisitions were performed at $80 \mathrm{kVp}$. For FBP and 100\% ASIR reconstructions, the fitting curves, SD $\sim \mathrm{CTDI}^{-1 / 2}$ and SDNR $\sim \mathrm{CTDI}^{1 / 2}$, were added as guided for the eyes, while for VEO, fitting curves with a non-zero intercept $\left(\alpha+\mathrm{CTDI}_{\mathrm{vol}}^{ \pm 1 / 2}+\beta\right.$ where $\alpha$ and $\beta$ are constant parameters) were used. 

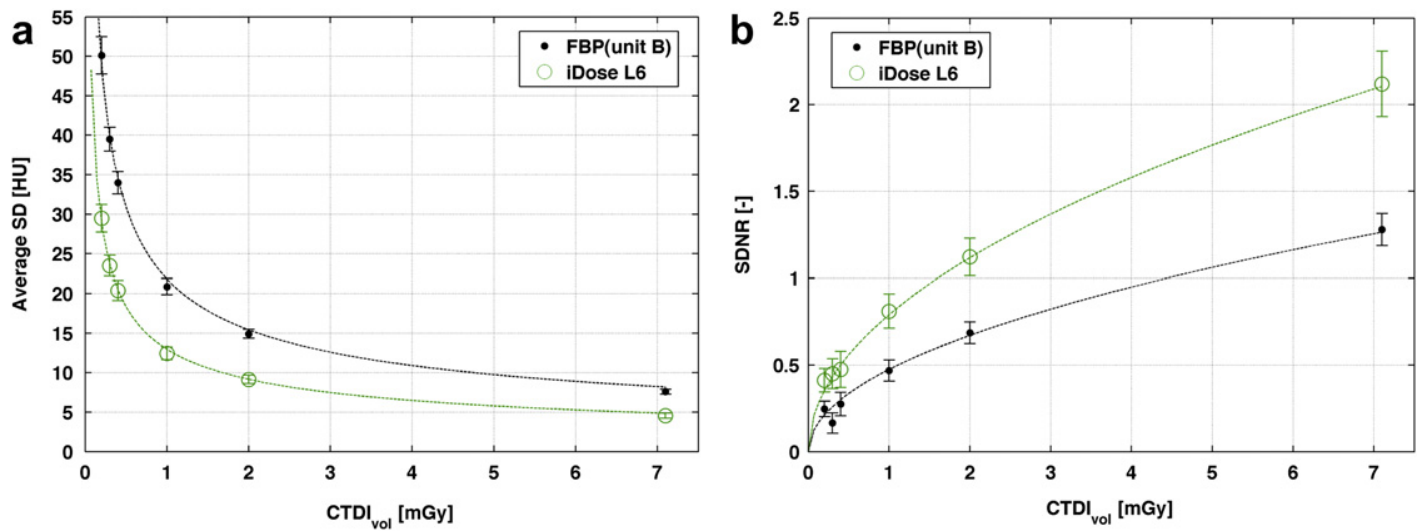

Figure 8 (a) Average SD and (b) SDNR measured on the low-contrast cylinder (Fig. 1a) as a function of dose on unit B. Acquisitions were performed at $80 \mathrm{kVp}$. The fitting curves, $\mathrm{SD} \sim \mathrm{CTDI}^{-1 / 2}$ and SDNR $\sim \mathrm{CTDI}^{1 / 2}$, were added as guided for the eyes.

that the important discrepancy in detectability between Figs. 11 and 12 is mainly attributed to partial volume effect produced by the small bead size.

\section{Discussion}

The purpose of this work was to characterize and compare three iterative reconstruction methods available on two commercial CT units using physical metrics and 4AFC detectability experiments. Because protocol parameters were slightly different (due to CT unit specificities), results were analyzed only when compared to the conventional FBP reconstruction method within the same unit.

For unit $A$, the second-generation iterative reconstruction VEO showed a large reduction of image noise combined with an improvement of spatial resolution. At $0.5 \mathrm{mGy}$, up to $70.4 \%$ less noise was observed with VEO in comparison

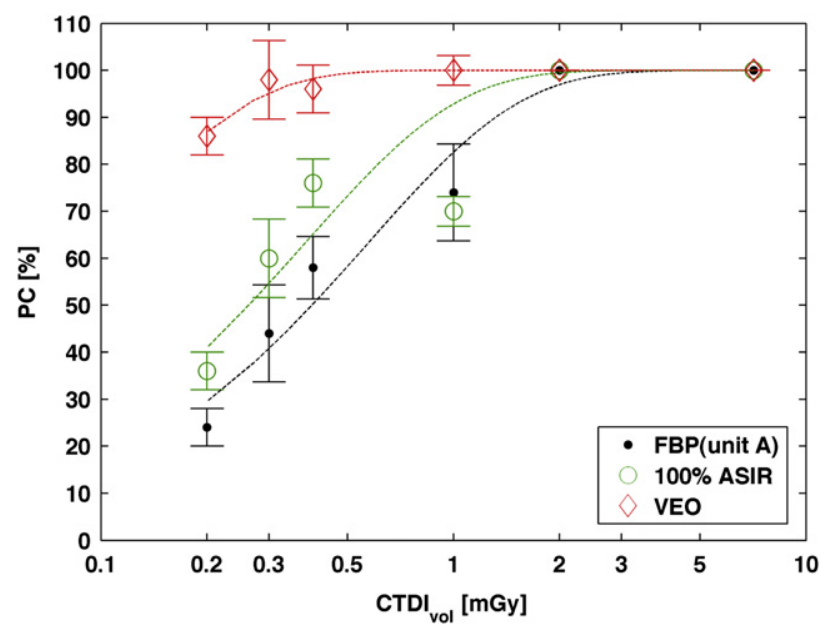

Figure 9 4AFC experiments performed for the low-contrast sphere object on unit A. Percent correct is expressed as a function of various doses for the three different reconstructions: FBP, $100 \%$ ASIR and VEO. Note that for better visibility, one-parameter fitting curve, $\mathrm{PC}=100 \cdot\left(1-e^{-\mathrm{a} \cdot \mathrm{CTDI}}\right)$, was added as guided for the eyes and $\mathrm{CTDI}_{\text {vol }}$ was represented on log scale. Acquisitions were performed at $80 \mathrm{kVp}$. with the standard FBP reconstruction method, while with $100 \%$ ASIR $46.5 \%$ was obtained. However, contrary to ASIR (first-generation iterative reconstruction) and FBP, VEO does not reduce noise proportionally to the classical relation $\left(\mathrm{CTDI}_{\mathrm{vol}}^{-1 / 2}\right.$ ) dictated by a Poisson distribution and allows a higher noise diminution when dose reduces. Analysis of the NPS curve obtained with VEO shows that the frequency components of noise are differently modified compared to ASIR and FBP, leading to a change in the curve shape and noise texture.

The 4AFC detectability experiments showed that the observed amount of lower noise and higher resolution was materialized by a better observer performance. For both experiments performed on unit $A$, the low-contrast sphere and the microcalcification were correctly located at ultralow-dose $\left(P C=100 \%\right.$ at $0.3 \mathrm{mGy}$ ) while a $\mathrm{CTDI}_{\mathrm{vol}}$ higher than $1.0 \mathrm{mGy}$ was necessary to obtain similar results with FBP and $100 \%$ ASIR. Objects that could not be detected with FBP or $100 \%$ ASIR were visible with VEO. Results show that without reducing the object detectability (constant PC), a decrease of dose by a factor 3 to $7(67 \%-86 \%)$ is possible.

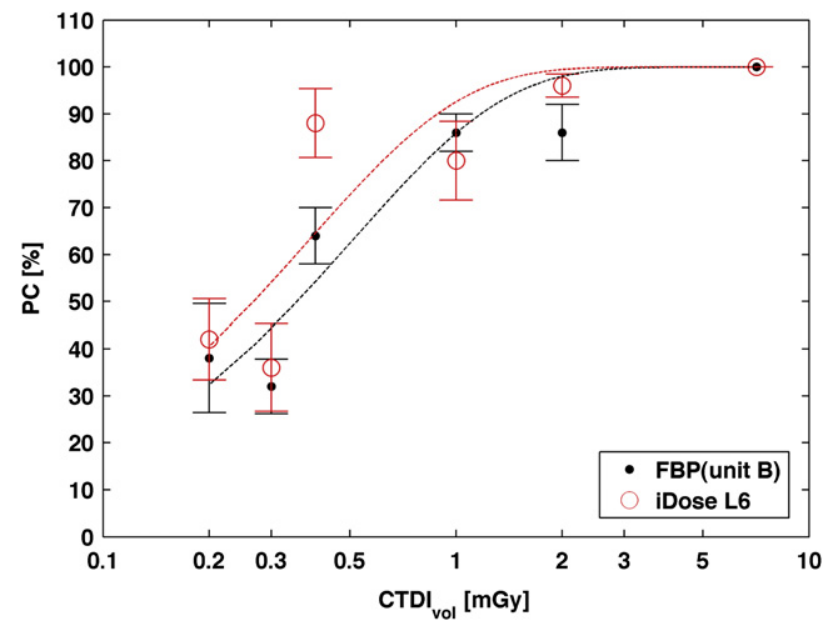

Figure $10 \quad 4 A F C$ experiment performed for the low-contrast sphere object on unit $B$. PC is expressed as a function of various doses for the two different reconstructions: FBP and iDose ${ }^{4}$ L6. Acquisitions were performed at $80 \mathrm{kVp}$. 


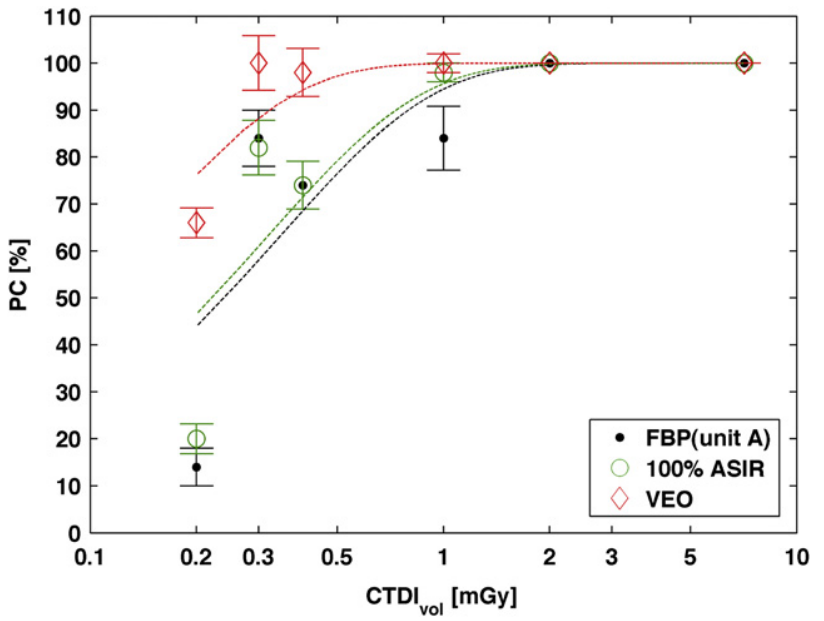

Figure 11 4AFC experiments performed for the microcalcification object on unit A. PC is expressed as a function of various dose for the three different reconstructions: FBP, $100 \%$ ASIR and VEO. Acquisitions were performed at $80 \mathrm{kVp}$.

However, although VEO seems to mark a major breakthrough in CT reconstruction, this complexity has a price. VEO requires a high computational power leading to a reconstruction time typically in the range of a few minutes to $1 \mathrm{~h}$, much longer than ASIR which only needs a few seconds for a large volume reconstruction. In spite of the fact that a faster reconstruction should become possible quickly as computing power increases, so far today, VEO is not yet suitable for all clinical situations.

For unit B, iDose ${ }^{4}$ provided an important reduction of SD metrics with few modifications of the NPS curves. As reported in [18], iDose ${ }^{4}$ attempts to reproduce the NPS of the FBP method to keep a noise texture that radiologists expect. However, it is worth pointing out that noise is reduced with no changes in MTF curves when compared to the FBP method. Thus, to obtain a higher spatial resolution, iDose $^{4}$ has to be conjugated with a sharper reconstruction

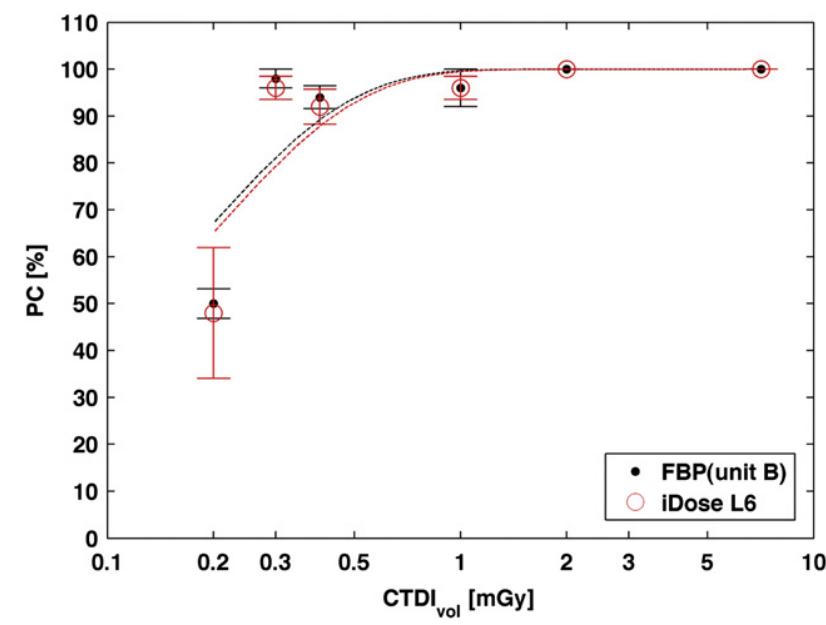

Figure 12 4AFC experiments performed for the microcalcification object on unit B. PC is expressed as a function of various doses for the two different reconstructions: FBP and iDose ${ }^{4}$ L6. Acquisitions were performed at $80 \mathrm{kVp}$. filter. This emphasizes the fact that to take full advantages of new iterative reconstructions and produce efficient lowdose $\mathrm{CT}$ protocols, a change in reconstruction parameters is required in addition to the iterative reconstruction method.

As reported in [28], the low-contrast task could be identified $100 \%$ of the time in the AFC experiments while SDNR values was less than the Rose criterion that suggests a threshold of $3-5$ for reliable $[29,30]$. In our study, $\mathrm{PC}=\sim 95 \%$ was achieved for SDNR values corresponding to $\sim 0.75$ with the FBP methods. This was attributed to observers who were able to detect the low-contrast sphere (in a 4AFC experiment and under the SKE paradigm) before it might be visible. Moreover, Rose criterion was established for white noise background. This assumption is not valid in CT where the NPS is low in the low-frequency range with a peak at midfrequences as shown in Figs. 5 and 6. Thus, for relatively large object as the $8 \mathrm{~mm}$ sphere, which corresponds to a low-frequency task function, the detectability is improved compared to detection in white noise.

From a physical point of view, iDose ${ }^{4}$ appears to be close to ASIR with reconstruction times similar to FBP for both techniques. However, the impact of a change in noise texture, which seems to play an important role when assessing diagnostic image quality (defined as the capacity of detecting a given pathology), was not considered in this study. Recently, several papers [11-14] investigated the use of first-generation iterative reconstructions in the clinic and reported a substantial change in image characteristics with potential lower diagnostic image conspicuity. It should be kept in mind that physical metrics only represent a fast and simple approach to evaluate some of the properties of reconstruction methods and may have limitations (especially for MTF measurements with non-linear reconstructions). Although SDNR show similitude with 4AFC results, the approach of linking metrics to observer performance is complex and thresholds for detectability limits stay difficult to establish on the basis of simple metrics. Detection experiments performed on phantoms, as we performed in this study, or even better, clinical studies performed on patient population cohorts, with diagnostic image quality evaluated by radiologists on receiver operating characteristic (ROC)-based approaches, remain the gold standard. Today, understanding if the noise texture of iterative reconstructions can affect the diagnostic quality for a patient or if it is only a matter of habit due to radiologist background (used to read FBP images) appears to be an important challenge to safely set up low- and ultra-lowdose protocols.

\section{Conclusion}

Based on phantom approaches, our results show that firstgeneration iterative reconstruction methods ASIR and iDose ${ }^{4}$ provide similar benefits and mainly act on noise reduction. Clinical studies have confirmed that these methods are able to strongly reduce image noise and allow a significant dose reduction (30-65\%) with reconstruction time almost as fast as standard FBP methods [11-14,19]. Second-generation iterative reconstruction VEO shows extra noise reduction when compared to first-generation iterative methods as well as a significant spatial 
resolution improvement. This was corroborated by the 4AFC detection experiments performed on phantoms, and suggests a potential dose reduction of factor 3 to 7 (67\%$86 \%)$. Because of long computer processing times, VEO appears to be practically useful for patient follow-up receiving significant doses as pediatric patients where cumulative lifetime dose still remains high. However, in order to fully evaluate the potential of VEO for dose reduction, further clinical studies are necessary to determine the influence of new VEO image texture on diagnostic conspicuity.

\section{Acknowledgments}

We would like to thank Professor Olivier Hélénon, chairman of the adult radiology department in the Necker Children's Hospital of Paris, for his assistance and for providing the machine time required to perform this study as well as Stéphanie Bermon, Ivan Diaz, Eleni Samara and Dimitra Touli, for their participation in this study. This research was supported by the Swiss National Science Foundation grant [\# 320030-120382].

\section{References}

[1] BFS. Umweltradioaktivität und Strahlenbelastung im Jahr 2003. Bundesministerium für Umwelt, Naturschutz und Reaktorsicherheit (BMU); 2003. Unterrichtung durch die Bundesregierung (Parlamentsbericht), Berichtjahr.

[2] Brix G, Nagel H, Stamm G, Veit R, Lechel U, Griebel J, et al. Radiation exposure in multi-slice versus single-slice spiral $\mathrm{CT}$ : results of a nationwide survey. Eur Radiol 2003;13:1979-91.

[3] Aroua A, Vader JP, Valley JF, Verdun FR. Exposure of the Swiss population by radiodiagnostics: 2003 review. Health Phys 2007;92:442-8.

[4] Shrimpton C, Hillier MC, Lewis MA, Dunn M. National survey of doses from CT in the UK: 2003. Br J Radiol 2006;79:968-80.

[5] Mettler Jr FA, Thomadsen BR, Bhargavan M, Gilley DB, Gray JE, Lipoti JA, et al. Medical radiation exposure in the U.S. in 2006: preliminary results. Health Phys 2008;95:502-7.

[6] UNSCEAR. Sources and effects of ionizing radiation. United Nations Scientific Committee on the Effects of Atomic Radiation 2000 Report to the General Assembly, with Scientific Annexes. volume I: Sources. UN Sales Publication E.00.IX.3. New York, USA: United Nations, 2000.

[7] Kalender WA, Buchenau S, Deak P, Kellermeier M, Langner O, van Straten $M$, et al. Technical approaches to the optimization of CT. Phys Med 2008;24:71-9.

[8] McCollough CH, Bruesewitz MR, Kolfer JM. CT dose reduction and dose management tools: overview of available options. Radiographics 2006;26:503-12.

[9] Gunn MLD, Kohr JR. State of the art: technologies for computed tomography dose reduction. Emerg Radiol 2009;17:209-18.

[10] Ulzheimer S, Endt H, Flohr T. Computed tomography-patient dose and dose reduction technologies. Health Phys 2011; 100(3):325-8.

[11] Hara AK, Paden RG, Silva AC, Kujak JL, Lawder HJ, Pavlicek W. Iterative reconstruction technique for reducing body radiation dose at CT: feasibility study. AJR 2009;193:764-71.

[12] Silva AC, Lawder HJ, Hara A, Kujak J, Pavlicek W. Innovations in CT dose reduction strategy: application of the adaptive statistical iterative reconstruction algorithm. AJR 2010;194:191-9.
[13] Marin D, Nelson RC, Schindera ST, Richard S, Youngblood RS, Yoshizumi TT, et al. Low-tube-voltage, high-tube-current multidetector abdominal CT: improved image quality and decreased radiation dose with adaptive statistical iterative reconstruction algorithm - initial clinical experience. Radiology 2010;254:145-53.

[14] Miéville FA, Gudinchet F, Rizzo E, Ou P, Brunelle F, Bochud FO, et al. Paediatric cardiac CT examinations: impact of the iterative reconstruction method ASIR on image quality preliminary findings. Ped Radio; 2011 Jun 30 [Epub ahead of print].

[15] Ghetti C, Ortenzia O, Serreli G. CT iterative reconstruction in image space: a phantom study. Physica Med; 2011 Apr 14 [Epub ahead of print].

[16] Pontana F, Pagniez J, Flohr T, Faivre JB, Duhamel A, Remy J, et al. Chest computed tomography using iterative reconstruction vs filtered back projection (part 1): evaluation of image noise reduction in 32 patients. Eur Radiol 2011;21:627-35.

[17] Pontana F, Duhamel A, Pagniez J, Flohr T, Faivre JB, Hachulla $\mathrm{AL}$, et al. Chest computed tomography using iterative reconstruction vs filtered back projection (part 2): image quality of low-dose CT examinations in 80 patients.

[18] Fleischmann D, Boas FE. Computed tomography-old ideas and new technology. Eur Radiol 2011;21(3):510-7.

[19] Yoshinori F, Katsuyuki T, Daisuke U, Seitaro O, Yumi Y, Yasuyuki $Y$, et al. Combination of a low-tube-voltage technique with hybrid iterative reconstruction (iDose) algorithm at coronary computed tomographic angiography. J Comput Assist Tomogr 2011;35(4):480-5.

[20] Iterative reconstruction workshop session at SPIE Medical Imaging. Orlando; 2011.

[21] Yadava G, Kulkarni S, Colon ZR, Thibault J, Hsieh J. TU-A201B-03: Dose reduction and image quality benefits using model based iterative reconstruction (MBIR) technique for computed tomography. AAPM Annual Meeting (2010), 3372.

[22] International Electrotechnical Committee. Medical diagnostic $X$-ray equipment - radiation conditions for use in the determination of characteristics; 1994. Standard IEC 61267, Geneva.

[23] International Electrotechnical Committee. Medical diagnostic $X$-ray equipment - particular requirements for the safety of X-ray equipment for CT; 1999. Standard IEC 60601-2-44, Geneva.

[24] International Electrotechnical Committee. Medical diagnostic $X$-ray equipment - particular requirements for the safety of X-ray equipment for CT. 2nd ed.; 2002. Standard IEC 60601-244, Geneva.

[25] JCGM 100:2008. Evaluation of measurement data - guide to the expression of uncertainty in measurement. 1st ed.; September 2008.

[26] Miéville FA, Ayestaran P, Argaud C, Rizzo E, Ou P, Brunelle F, et al. Potential benefit of the CT adaptive statistical iterative reconstruction method for pediatric cardiac diagnosis. Proc SPIE 2010;7622. pp. 76222D1-76222D11.

[27] Yang K, Kwan ALC, Huang SY, Packard NJ, Boone JM. Noise power properties of a cone-beam CT system for breast cancer detection. Med Phys 2008;35(12):5317-27.

[28] Tward DJ, Siewerdsen JH, Daly MJ, Richard S, Moseley DJ, Jaffray DA, et al. Soft-tissue detectability in cone-beam CT: evaluation by $2 \mathrm{AFC}$ tests in relation to physical performance metrics. Med Phys 2007;34(11):4459-71.

[29] Rose A. Quantum effects in human vision. Adv Biol Med Phys 1957;5:211-42.

[30] Burgess AE. The Rose model, revisited. J Opt Soc Am A 1999; 16(3):633-46. 